DOI 10.37882/2223-2982.2020.05.04

\title{
О КОГНИТИВНО-ДИНАМИЧЕСКОЙ КОНЦЕПЦИИ СИНХРОННОГО ПЕРЕВОДА
}

\section{ON COGNITIVE DYNAMIC CONCEPT OF SIMULTANEOUS INTERPRETATION \\ D. Balaganov}

Summary: This article is devoted to process-oriented aspects of the simultaneous interpretation. The author provides his cognitive dynamic concept that characterizes the work of the cognitive mechanisms and stress factors that affect them. The article contains the views of the author on the reasons that change the functioning of the cognitive mechanisms. In conclusion, the author proposes the ways to increase the stress resilience of the cognitive system of the simultaneous interpretation.

Keywords: simultaneous interpretation, cognitive dynamic concept, stress factors, stress resilience, cognitive mechanism, cognitive system.

\author{
Балаганов Дмитрий Владимирович \\ К.филол.н., Военный университет Министерства \\ обороны Российской Федерации, Москва \\ dmitryrus@yandex.ru
}

Аннотация: Настоящая статья посвящена процессуальным аспектам синхронного перевода. Автор представляет выдвигаемую им когнитивно-динамическую концепцию, характеризующую работу когнитивных механизмов и влияющих на них стрессогенных факторов. Статья содержит взгляды автора на причины изменений, происходящих в функционировании тех или иных когнитивных механизмов. В заключение автор предлагает пути повышения стрессоустойчивости когнитивной системы синхронного перевода.

Ключевые слова: синхронный перевод, когнитивно-динамическая концепция, стрессогенные факторы, стрессоустойчивость, когнитивный механизм, когнитивная система.

\section{BBEAEHИE}

роцессуальный подход к проблемам синхронного перевода все чаще привлекает различных отечественных и зарубежных ученых [8, 9, 10, 13]. Некоторые из них предпринимают попытки исследовать его с когнитивной точки зрения. Практика синхронного перевода показывает, что переводчик-синхронист неравномерно осуществляет акт перевода. На него постоянно оказывают влияния различные факторы. Однако комплексных исследований всех аспектов синхронного перевода как процесса не проводилось уже почти полвека. Этим обусловливается актуальность нашей научной работы.

Проведенные нами ранее теоретические и эмпирические исследования, а также собственный практический опыт работы в области синхронного перевода позволил впервые предложить когнитивно-динамический подход к синхронно-переводческой деятельности. В этом заключается новизна нашей статьи.

Целью нашей публикации является представить собственную когнитивно-динамическую концепцию синхронного перевода. Для ее достижения нами выполнены следующие задачи:

- представить сущность когнитивно-динамической концепции и ее основных положений;

- описать когнитивную систему синхронного перевода;

- охарактеризовать динамику состояния языкового сознания переводчика в процессе синхронного перевода;
- определить пути повышения стрессоустойчивости когнитивной системы синхронного перевода.

Результаты нашего исследования могут быть использованы для дальнейшего изучения процессуальных аспектов синхронного перевода, а также для учета при организации образовательного процесса подготовки синхронно-переводческих кадров.

\section{ОСНОВНЫЕ ПОЛОЖКЕНИЯ КОГНИТИВНО-АИНАМИЧЕСКОЙ КОНШЕПЧИИ}

Когнитивно-динамическая концепция синхронного перевода описывает его процесс как когнитивную систему, которая состоит из различных когнитивных механизмов. Когнитивные механизмы в нашем понимании представляют собой совокупность когнитивных операций, обеспечивающих выполнение механизмами своей части когнитивных задач в процессе синхронного перевода [3]. Каждый когнитивный механизм функционирует таким образом, чтобы обеспечивать работу других когнитивных механизмов системы синхронного перевода, дополняя и уточняя поступающую информацию. К числу когнитивных механизмов синхронного перевода мы относим следующие: восприятия и понимания, обработки информации, переключения между языками, выбора варианта перевода, вероятностного прогнозирования, синхронизации. Подробнее о каждом когнитивном механизме остановимся в следующем разделе.

В процессе синхронного перевода, помимо естественного стресса, вызванного необычностью когни- 
тивной задачи, при которой переводчик вынужден выполнять несколько когнитивных операций практически одновременно, на синхрониста могут влиять и дополнительные стрессогенные факторы, как объективного, так и субъективного характера. При этом языковое сознание переводчика-синхрониста подвергается в той или иной степени изменению. В связи с этим его способность выполнять синхронно-переводческую задачу также меняется. В данном случае важно отметить, что от того, насколько быстро переводчик может адаптироваться к новым условиям работы или восстановить неизмененное состояние своего языкового сознания, зависит его профессиональная надежность. Способы ее повышения будут рассмотрены нами ниже.

С учетом выше изложенного в рамках нашей когнитивно-динамической концепции мы постулируем следующие основные положения:

1. Процесс синхронного перевода представляет собой когнитивную систему, состоящую из когнитивных механизмов, которые обеспечивают выполнение синхронно-переводческой задачи. Результат работы каждого из когнитивных механизмов существенным образом влияет на функционирование других механизмов.

2. В процессе синхронного перевода переводчиксинхронист испытывает различную стрессогенную нагрузку. Она связана, с одной стороны, с тем, что переводчик вынужден работать в условиях острого дефицита времени и выполнять несколько когнитивных операций одновременно; с другой стороны, такая нагрузка может возникать вследствие различных субъективных и объективных факторов и оказывать существенное влияние на профессиональную надежность переводчика.

3. Стрессогенные факторы различного генеза могут приводить к изменению состояния языкового сознания переводчика-синхрониста, в результате чего может произойти частичная или полная утрата им способности к выполнению синхроннопереводческой задачи. Адаптация синхронного переводчика к новым условиям работы, а также восстановления нормального состояния своего языкового сознания зависит от наработанных им приемов и подходов к нивелированию негативного влияния стресса.

Ниже нами будут подробнее рассмотрены каждые из этих положений.

\section{КОГНИТИВНАЯ СИСТЕМА СИНХРОННОГО

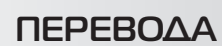

Когнитивная система синхронного перевода состоит из когнитивных механизмов, реализующих те или иные когнитивные задачи в интересах выполнения всей син- хронно-переводческой деятельности.

Когнитивные механизмы, в свою очередь, включают в себя когнитивные операции, необходимые для их функционирования в системе. Они представляют собой автоматизированные действия, выполняемые на подсознательном уровне по определенной схеме. Отработка таких когнитивных операций позволяет переводчикусинхронисту снизить когнитивную нагрузку в ходе синхронного перевода.

Когнитивный механизм восприятия и понимания отвечает за распознавание поступающих фонем, сопоставление их с хранящимися в памяти образцами и последующее соединение их в лексемы. На данном этапе не происходит вычленение смысловой нагрузки речи оратора, а всего лишь возникает образ слов. Другими словами, происходит декодирование поступающей звуковой информации.

Когнитивный механизм обработки информации реализует задачи, связанные с установлением синтаксической связи в предложении, определении принадлежности той или иной лексемы к определенной части речи (в случае омонимии), а также выявлении ее значения в зависимости от лингвистического и/или экстралингвистического контекста. Данный когнитивный механизм также занимается вопросами смыслового осмысления услышанной информации.

Когнитивный механизм переключения между языками удерживает языковые системы двух языков (исходного языка и языка перевода) в активном состоянии после их активации в начале акта синхронного перевода [7]. Происходит этого вследствие постоянной необходимости воспринимать информацию на одном языке и сопоставлять возможные способы ее передачи в системе языка перевода.

Когнитивный механизм выбора варианта перевода отвечает за сканирование языковой системы языка перевода на предмет наилучшего способа передачи смысла поступающей от оратора информации. На начальном этапе синхронно-переводческой деятельности переводчик использует, в основном, знаковый способ перевода. Однако, по мере накопления информации относительно интенции оратора, он может выполнять определенную смысловую обработку услышанного. В связи с этим выбор наилучшего варианта перевода упрощается. По завершении работы данного когнитивного механизма переводчик вербализует выбранный вариант перевода.

Помимо указанных выше существуют еще два когнитивных механизма: вероятностного прогнозирования и синхронизации. Они располагаются как бы над всеми ними и обеспечивают их функционирование. 
Когнитивный механизм вероятностного прогнозирования работает на основе лингвистического и/или экстралингвистического контекста (фоновых знаний переводчика). Его сущность заключается в способности переводчика-синхрониста догадываться о возможном окончании высказывания еще до его завершения. При этом он опирается на лингвистического окружение, которое может указывать на конкретное построение выражения (в случае, например, с фразеологизмом) или на имеющиеся у него фоновые знания, которые он накопил в преддверии акта синхронного перевода или при работе по одной и той же тематике. В этом переводчику также помогает кумулятивно-динамический анализ, то есть осмысление через поступившую речь оратора его интенции.

Когнитивный механизм синхронизации обеспечивает работу всех когнитивных механизмов. Поэтому он является системообразующим и необходимым элементом всей когнитивной системы. Он управляет вниманием переводчика с тем, чтобы оно было направлено на наиболее трудоемкие элементы воспринимаемого исходного текста. Обычно при переводе с иностранного языка на родной основное внимание уделяется речи оратора, поскольку система родного языка затрачивает меньше когнитивных усилий. Однако даже и в этом случае бывают ситуации, когда сказанное оратором понятно переводчику, но как лучше передать услышанное на родном языке вызывает определенную трудность. В частности, такие сложности возникают вследствие разного лексического объема тех или иных слов и выражений, а также при переводе безэквивалентной лексики.

Таким образом, когнитивная система синхронного перевода представляет собой стройную структуру, обеспечивающую выполнение переводчиком синхроннопереводческой деятельности.

\section{АИНАМИКА СОСТОЯНИЯ ЯЗЫКОВОГО СОЗНАНИЯ}

Если не учитывать такие субъективные факторы, которые влияют на работу переводчика, как недостаточный сон и психологические проблемы (их переводчик может избежать в большинстве случаев), то существуют объективные стрессогенные факторы, не зависящие от переводчика. К числу таких факторов можно отнести произносительные особенности и темп речи оратора (осложняют восприятие и понимание), использование узкоспециальной терминологии и аббревиатур (влияет на обработку информации), межъязыковая В ходе выполнения задач по обеспечению синхронного перевода на синхрониста оказывают влияние различные стрессогенные факторы. Поскольку синхронный перевод является когнитивной задачей повышенной сложности, при которой переводчику приходится задействовать не- сколько когнитивных операций одновременно, то данный вид перевода сам по себе обладает высокой стрессогенностью. Однако переводчик-синхронист, готовясь к акту синхронного перевода, постоянно готовит себя к работе в условиях такой ситуации перевода.

Тем не менее, ни переводчик, ни организатор мероприятия не всегда могут предсказать возможное развитие событий интерференция вследствие нестандартного произношения и других причин (затрудняет переключение между языками), а также вызванное ей нарушение норм языка перевода (влияет на выбор варианта перевода). Перечисленные факторы также оказывают негативное влияние на когнитивные механизмы вероятностного прогнозирования и синхронизации, не позволяя им выполнять свои функции.

Помимо указанных выше факторов, относящихся к системе синхронного перевода, существует еще ряд факторов внешнего характера. Среди них отметим температуру и качество воздуха в кабине синхронного перевода, а также техническую готовность аппаратуры (подача аудио- и видеосигнала для обеспечения мультиканальности восприятия исходного текста $[6,12])$.

При нарушении функционирования одного из когнитивных механизмов по причине негативного влияния стрессогенных факторов расстраивается вся система синхронного перевода. При этом происходит изменение состояния языкового сознания переводчика [2], что может привести к различным искажениям при передаче речи оратора. Для подтверждения данного тезиса нами был проведен психолингвистический и психофизиологический эксперимент [1].

В результате такого изменения сознания переводчик частично или полностью теряет способность адекватно выполнять синхронно-переводческую задачу. В зависимости от опыта и психологической устойчивости к стрессу (стрессоустойчивости) [5] переводчику требуется разное время на адаптацию к новым условиям работы или для восстановления нормального состояния своего языкового сознания.

Находясь в состоянии стресса, при которой его способность к осуществлению переводческой задачи снижена, переводчик-синхронист задействует те или иные стратегии для решения возникающих у него переводческих и психологических проблем. В этой связи возникает вопрос предела допустимости опущения или интерпретации поступающей от оратора информации [4]. С одной стороны, опущение несущественной или избыточной информации и интерпретация помогают синхронному переводчику справится с трудностями при переводе. С другой стороны, как определить несущественность информации, а также до какой степени 
переводчику позволено самостоятельно ее интерпретировать без потери или искажения смысла высказывания?

\section{СТРЕССОУСТОЙЧИВОСТЬ СИСТЕМЫ}

Обычно термин «стрессоустойчивость» применяется исключительно к человеку как субъекту когнитивной деятельности. Однако практика показывает, что устойчивостью к стрессу обладают и различные системы, состоящие из взаимозависящих элементов, без которых функционирование всей системы невозможно (например, правоохранительная система государства). В нашем случае мы имеем в виду систему когнитивных механизмов синхронного перевода. С одной стороны, данная система включает в себя элементы, суммарное функционирование которых делает возможным процесс синхронного перевода. С другой стороны, когнитивная система синхронного перевода является одним из элементов человеческой психики. Поэтому мы полагаем, что использование термина «стрессоустойчивость» для нее вполне приемлемо.

Для обеспечения стрессоустойчивости когнитивной системы синхронного перевода необходимо выполнение двух условий: а) языковая подготовленность индивида к акту синхронного перевода и б) его психологическая готовность к непредвиденным изменениям в ситуации перевода.

Языковая составляющая стрессоустойчивости когнитивной системы синхронного перевода затрагивает все аспекты лингвистического компонента синхронно-переводческой деятельности. К ним мы относим совершенствование знания рабочих языков, постоянное отслеживание изменений в их лексическом составе, глубокое изучение тематики предстоящего мероприятия, знакомство с произносительными особенностями ораторов (по возможности), их намерениями и целями выступления. Помимо этого, важно постоянно отрабатывать различные приемы и стратегии для преодоления тех или иных трудностей перевода.

Психологический компонент стрессоустойчивости не менее важен. Одного только знания языка и терминологии недостаточно для успешного перевода. Наш собственный опыт работы в качестве синхронного переводчика в интересах официальных лиц различного уровня показывает, что отсутствие готовности оперативно реагировать на возникающие внешние трудности перевода вполне может привести к потере значительной информации. Психологическая готовность может тренироваться при помощи аппаратных (специальные приборы для выбранной настройки ритмов головного мозга, в том числе и методика биологической обратной связи) и неаппаратных (звуковое, цветовое и вкусовое воздействие для настройки головного мозга) средств. Наиболее оптимальное сочетание подбирается индивидуально в зависимости от психологических особенностей личности.

При правильной тренировке стрессоустойчивости когнитивная система синхронного перевода будет менее подвержена негативному влиянию стресса, проявляя определенную гибкость [11]. Она будет обеспечивать адекватное функционирование всех механизмов для успешного выполнения синхронно-переводческой задачи.

\section{выво $\triangle \mathrm{b}$}

В результате проведенного нами исследования мы пришли к следующим выводам:

1. Когнитивная система синхронного перевода состоит из когнитивных механизмов восприятия и понимания, обработки поступающей информации, переключения между языками, выбора варианта перевода, вероятностного прогнозирования и синхронизации. Эта система обеспечивает выполнение переводчиком синхронно-переводческой задачи. Все механизмы внутри системы взаимосвязаны. Любое нарушение в работе одного из них ведет к разбалансировке всей системы.

2. В процессе синхронного перевода на индивида оказывают негативное влияние различные стрессогенные факторы, как субъективного, так и объективного характера. В результате этого может происходить изменение состояния языкового сознания переводчика, что в той или иной мере снижает его способность осуществлять адекватный перевод. В зависимости от степени стрессоустойчивости всей когнитивной системы синхронного перевода переводчик-синхронист адаптируется к новым условиям работы или восстанавливает неизмененное состояние своего языкового сознания.

3. Для повышения стрессоустойчивости когнитивной системы синхронного перевода необходимо осуществлять подготовку по двум направлениям: лингвистическому и психологическому. При этом психологический тренинг подбирается индивидуально для каждого переводчика. Степень всесторонней готовности переводчика к акту синхронного перевода в меняющихся условиях ситуации перевода влияет на его профессиональную надежность.

\section{ЗАКАЮЧЕНИЕ}

В настоящей публикации нами была представлена когнитивно-динамическая концепция синхронного перевода. Она позволяет взглянуть на деятельность синхронного переводчика с точки зрения функционирова- 
ния его когнитивных механизмов, предназначенных для выполнения конкретной переводческой задачи.

Синхронный перевод представляется нам как динамическая когнитивная деятельность, обладающая определенной гибкостью и требующая от переводчика быстрого принятия решения относительно возникающих переводческих и непереводческих трудностей с тем, чтобы минимизировать последствия негативного влияния стрессогенных факторов. Поэтому анализ функцио- нирования когнитивных механизмов, обеспечивающих данный вид перевода, крайне важен для всестороннего понимания процессов, действующих при работе переводчика-синхрониста.

Результаты нашего исследования могут быть использованы для дальнейшего теоретического изучения процессуальных аспектов синхронного перевода, а также учтены при организации образовательного процесса по подготовке синхронно-переводческих кадров.

\section{ЛИТЕРАТУРА}

1. Балаганов Д.В. Влияние стресса на деятельность переводчика-синхрониста // Филологические науки. Вопросы теории и практики. №12 (90). Ч.1. М.: Грамота, 2018. С.74-79.

2. Балаганов Д.В. Динамика состояния языкового сознания индивида в процессе синхронного перевода // Вестник МгоУ. Серия: Лингвистика. №1. Москва: МГОУ, 2020. С.6-14.

3. Балаганов Д.В., Давыдова Т.Ю. Когнитивные механизмы в синхронном переводе // Вестник Нижегородского государственного лингвистического университета им. Н. А. Добролюбова. №47. Нижний Новгород: ННГЛУ, 2019. С.19-32.

4. Иванов Н.В. Интерпретация в знаковой онтологии языка и в переводе: монография /Н.В. Иванов. Москва: ИД «Международные отношения», 2018. 152 с.

5. Конина А.А., Черниговская Т.В. Синхронный перевод как экстремальный вид когнитивных процессов (обзор экспериментальных исследований) // Вопросы психолингвистики. 2018. № 38. С.178-204.

6. Чистова Е.В. Мультиканальность и мультимодальность восприятия в когнитивной деятельности переводчика-синхрониста // Филологические науки. Вопросы теории и практики. Т.12. Вып.9. Тамбов: Грамота, 2019. С.337-342.

7. Aparicio, X., Heidlmayr, K., \& Isel, F. (2017). Inhibition Efficiency in Highly Proficient Bilinguals and Simultaneous Interpreters: Evidence from Language Switching and Stroop Tasks // Journal of Psycholinguistic Research. V.46, Issue 6. P.1427-1451.

8. Camayd-Freixas, E. (2011). Cognitive theory of simultaneous interpreting and training // Proceedings of the 52nd Conference of the American Translators Association. New York: ATA.

9. Chesterman, A. (2015). Models of What Processes? // Describing Cognitive Processes in Translation / ed. by M. Ehrensberger-Dow et al. Amsterdam, PA: Benjamins. P.7-20.

10. Gile D. (2018). Simultaneous Interpreting // CHAN, Sin-wai (ed). 2018. An Encyclopedia of Practical Translation and Interpreting. Hong Kong: The Chinese University Press. P.531-561.

11. Hervais-Adelman A., Moser-Mercer B., Golestani N. (2015). Brain Functional Plasticity Associated with the Emergence of Expertise in Extreme Language Control // Neuroimage. № 114. P.264-274.

12. Seeber, K.G. (2017). Multimodal Processing in Simultaneous Interpreting // The Handbook of Translation and Cognition. John Wiley \& Sons, Inc. P.461-475.

13. Setton, R. (1999). Simultaneous Interpretation: A Cognitive-Pragmatic Analysis. Amsterdam, PA: Benjamins. 399 p.

(с Балаганов Дмитрий Владимирович (dmitryrus@yandex.ru). 